Article

\title{
Automatic Vegetation Identification and Building Detection from a Single Nadir Aerial Image
}

\section{Nicholas Shorter * and Takis Kasparis}

School of Electrical Engineering and Computer Science (EECS), University of Central Florida (UCF), 4000 Central Florida Blvd., Orlando, FL 32816-2362, USA; E-Mail: kasparis@ucf.edu

* Author to whom correspondence should be addressed; E-Mail: nshorter@mail.ucf.edu; Tel.: +1-407-230-0452; Fax: +1-407-823-5835.

Received: 18 September 2009; in revised form: 30 September 2009 / Accepted: 13 October 2009 / Published: 16 October 2009

\begin{abstract}
A novel, automatic tertiary classifier is proposed for identifying vegetation, building and non-building objects from a single nadir aerial image. The method is unsupervised, that is, no parameter adjustment is done during the algorithm's execution. The only assumption the algorithm makes about the building structures is that they have convex rooftop sections. Results are provided for two different actual data sets.
\end{abstract}

Keywords: vegetation identification; building detection; color invariants; nadir aerial image

\section{Introduction}

Although overlapping geospatial information from multiple sources is becoming more and more available (LiDAR, multispectral, and stereo pair aerial imagery), many areas still exist with coverage from only a single nadir aerial image. A nadir, aerial image is defined as an image taken from the perspective of the sky overhead looking towards the Earth and taken at a high enough altitude such that there is only minute geometric distortion. Applications for automatic building detection from aerial imagery include geographic information system (GIS) database and map updating, change detection, target recognition, urban planning and site modeling.

Even with the increasing resolution of newer sensors to capture overhead aerial imagery, several factors still present challenges which hinder perfect building detection. These factors, as reported in [1], include the scene complexity, building variability, and sensor resolution. Another factor is overlapping multiple sensor coverage of the same scene: fusing different sensor data together could reduce the 
difficulty of detecting buildings for that scene. Rottensteiner et al. in [2,3] and Vosselman et al. in [4] make use of both Light Detection and Ranging (LiDAR) data and a single nadir, aerial image for automatic building detection and vegetation identification. Other approaches such as those benchmarked in the PRRS 2008 contest [5] and Jin and Davis in [6] make use of multispectral imagery.

Most building detection from aerial imagery approaches can be classified according to whether they are automatic or supervised (require a training phase) and whether they extract geometric features such as lines, corners, etc. or are area based. There are of course exceptions and some hybrid methods employ both geometric features and areas or have both automatic phases and semiautomatic supervised phases. Lefevere et al. in [7] propose an area based automatic building detection from aerial image approach which employs morphological filtering. First, binary images are created by clustering the aerial image's grey scale histogram. Then, multiple clusters are fused together and added to the original set of binary images. Finally, morphological opening, followed by the hit or miss transform and then geodesic reconstruction are performed for building detection. Their approach realized a pixel level completeness of $63.6 \%$ and a pixel level correctness of $79.4 \%$ (no building level completeness or correctness were reported). While the authors approach is automatic, it unfortunately implements the assumption that buildings are square or rectangular. The authors compute a two-dimensional granulometry of the binary images varying the width and length of the rectangular window. Because they do not vary the orientation of the window, they assume the rectilinear buildings are all parallel with one another and that the image has been rotated so that the sides of the buildings are parallel with the edges of the image.

Muller et al. in [8] implement an area and feature based algorithm for building detection from aerial imagery. The image is converted to grayscale and then segmented with a region growing algorithm. Then both photometric and geometric features are detected in each segmented region. Finally, a linear regression classifier then identifies building regions based on the extracted features. Their method is automatic and only takes 45 to 75 minutes on a $6,400 \times 6,400$ pixel image. Unfortunately, they implement the assumption that building rooftop hues primarily exist in the red channel of the RGB image. Furthermore, of the results pictorially presented in their paper for the 240 tested buildings (most of which have red roof tops), their algorithm had trouble correctly identifying several of the non-red roof top buildings. For the data sets their algorithm was tested on, the authors report a mean completeness and correctness for $79.5 \%$ and $78.5 \%$ (respectively).

Persson et al in [9] implement a supervised approach for building detection using an ensemble of self organizing maps (ESOM). Then, using the Hue Saturation Value (HSV) representation of the color aerial image, the ESOM is trained to recognize red roofs, light roofs, dark roofs and copper roofs. Rectangles are detected and then classified by ESOM as building or non-building. Their approach realizes a completeness and correctness of 53\% and 93\% respectively for a campus area. Because their approach is supervised, it requires a training phase. Furthermore, because only rectangles are classified as building/non-building, the approach assumes buildings are rectilinear. Results based on testing across 17 buildings are presented.

Sirmacek et al. in [10] have developed a feature and area based approach employing color invariant features [11] and shadow information for building detection from aerial imagery. Shadows are detected by Otsu thresholding [12] a blue color invariant image and red building roof tops are detected by thresholding the red color invariant image. They estimate the illumination direction by calculating the 
average direction between all the red roof top centroids and their adjacent shadow centroids. Then the illumination angle is used to find other non-red roof top regions by searching from the shadow region opposite of the illumination angle to nearby adjacent regions. A canny edge detector is run on the image and then a novel box fitting algorithm is grown within candidate building regions by minimizing an energy function. The inside of the rectilinear box is then assumed to be a building. The authors present results for only 177 buildings with a completeness of $86.6 \%$ (no correctness reported). Their approach assumes buildings are rectilinear and at least some portion of the data set contains red roofs. The illumination angle estimation is based on red roofs only and then is used to verify which adjacent region shadows have been cast from. Furthermore, their approach also assumes buildings are composed of either a single texture or a single roof plane. The canny edge detector will pick up edges where two textures or roof panels join and the box fitting algorithm will stop prematurely at the single roof panel adjacent to the shadow, not growing to accompany other adjoining panels of different color or texture.

Liu et al. in [13] use a feature and area based approach coupled with a probability function to identify building regions. Their algorithm starts out segmenting the image. Then a set of features (such as contour edges, shadow ratios, shape features, region entropy, etc.) are identified in each region. Then the probability function calculates the confidence value that the given region is in fact a region corresponding to building. Some of the parameters of the probability function are determined from a training set where buildings were manually identified. They report a completeness and correctness of $94.5 \%$ and $83.4 \%$ on a data set having 277 buildings. The authors notice a problem with shadows as they vary under different illumination directions, and they plan to extend their work by testing their approach on different imagery at various resolutions.

Benediktsson et al. in [14] propose a paradigm for classifying different objects in an aerial image. The approach includes three steps. First, a differential morphological profile (DMP) is built by using a collection of geodesic opening and closing operations of different size on a gray tone aerial image. Then, due to the large number of features contained in the DMP, feature extraction is applied. Finally, the third step consists of using a supervised neural network to classify the features extracted in the second step. The authors test their proposed method on two data sets, the first being a satellite image with $5 \mathrm{~m}$ pixel resolution covering Athens (Greece) and the second being a satellite image with $1 \mathrm{~m}$ pixel resolution covering Reyjavik (Iceland). In the first experiment, the authors trained the neural network with one third of the samples provided and used the rest for testing. However, in the second experiment, the authors trained the neural network using half the training samples and the rest for testing. The proposed method realized an overall average accuracy of $80 \%$ on the first data set and $95.1 \%$ on the second data set. Observe that a different proportion of training samples has to be used for each data set and that the algorithm has to be retrained for the different data sets.

Surveying the related literature, several common issues arrive in automatic and semi-automatic/supervised building detection approaches from aerial imagery. Some will make limiting assumptions which may reduce the ability of the algorithm when executed for data sets other than what were presented in the associated paper. Examples of such limiting assumptions would be buildings only having specific root top colors [8-10], buildings only existing at right angle corners and/or buildings having parallel sides [7,10]. Rather than saying all buildings have parallel sides or right angle corners, a more general assumption would be that buildings tend to have convex hull 
rooftop sections. Furthermore, many houses have a variety of different color roof tops (other than red) and commercial and industrial building roof tops include (but are not limited to) grey, black and white. Finally, the results should be tested on several hundred or even thousands of buildings of varying sizes, shapes, orientations, roof top colors, and roof top textures. Several of the current related papers in the literature [8-10,13] only present results from a couple of hundred buildings. While the semi-automatic/supervised approaches $[9,13,14]$ tend to have good accuracy, they rely on manually extracting features from $1 / 4$ to as much as $1 / 2$ the data set. Furthermore, unless an emphasis was placed on training the algorithm with data set invariant features, the algorithm will have to be retrained for each data set to achieve relatively close accuracy as what is presented in the associated paper. Furthermore, both completeness and correctness should be reported both on a global pixel level as well as a function of the building size.

We therefore propose an automated approach to building detection from a single, nadir, color aerial image with making very few assumptions about the buildings being detected. In striving to achieve automatic building detection, we have also come up with an automated approach for detecting vegetation as well from a single nadir, color aerial image. Results are presented for a given data set containing thousands of buildings of varying sizes, roof top colors and shapes. In Section 2, a detailed description of the proposed approach is presented. In Section 3, some characteristics about the two different data sets are described, evaluation metrics are discussed and results from the algorithm being tested on the two data sets are presented. In Section 4 we draw conclusions and future work is presented in Section 5.

\section{Algorithm Description}

The proposed building detection algorithm is an automatic region based approach and is implemented in four steps. In step 1 the input image is color segmented. In step 2 vegetation regions are identified and removed. In step 3 shadows are identified and removed and in step 4 the original color image is segmented again using an entropy segmentation algorithm. From these regions, non-building concrete surfaces such as parking lots, roads etc. are identified and removed leaving only building candidate regions. Each of the aforementioned steps are now described in detail in the subsequent subsections.

\subsection{Color Segmentation}

The vegetation detection algorithm relies on the following proposed color segmentation technique. The input image is color quantized, reducing the original 255 colors in each channel into 17, for a total of 51 values as each value in each channel is unique. Pixels in the red channel having intensities between 0 to 15 are represented by a single value, intensities 15 to 30 by another, intensities 30 to 45 by another, and so on and so forth. Each channel is then revisited and a single region label is assigned to all connected pixels (adjacent to one another via an eight-connected neighborhood) having the same quantized intensity values within the same channel. Region labels are assigned to connected groups of pixels for each of the three channels. Regions with less than 100 total pixels are discarded (marked with a common label and ignored in subsequent steps). All of the regions are then processed by a morphological closing operation, with a 5 by 5 square structuring element. Most of the roof panels in 
the smallest houses are larger than a 5 by 5 square. The 5 by 5 square is meant to eliminate thin region strands which typically do not belong to buildings. A single channel, color segmented image $O(i, j)$, where the intensity value $l_{o}$ at $\mathrm{i}, \mathrm{j}$ corresponds to the color segment that pixel belongs to, is then created by setting each pixel equal to the largest region label existent among the three channels for that pixel. At this point, single large regions that existed in the individual channels may be broken up in the single channel image. Therefore, the regions in the single channel image are discarded if they have less than 100 connected members. Then the single channel image is again processed by a morphological closing with a 7 by 7 square structuring element. The data sets being processed are on the order of 100 million pixels and therefore an efficient means of grouping the pixels based on similar color attributes was required. The aforementioned method was selected as it was able to process entire data sets on the order of minutes while producing acceptable results.

Figure 1. Input image and consolidated Output image. (a) Shows the subsection of the original aerial image; (b) Shows the largest region labels of the three channels; (c) Shows the final output after region labels with less than 100 pixels are removed and the image is processed by a morphological closing operator.

(a)

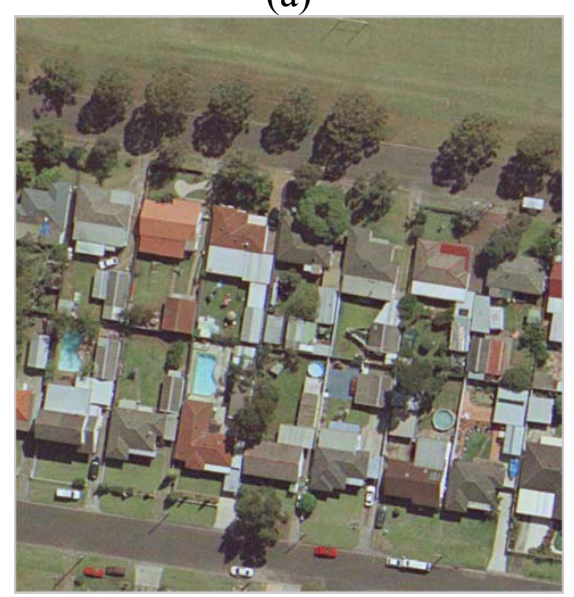

(b)

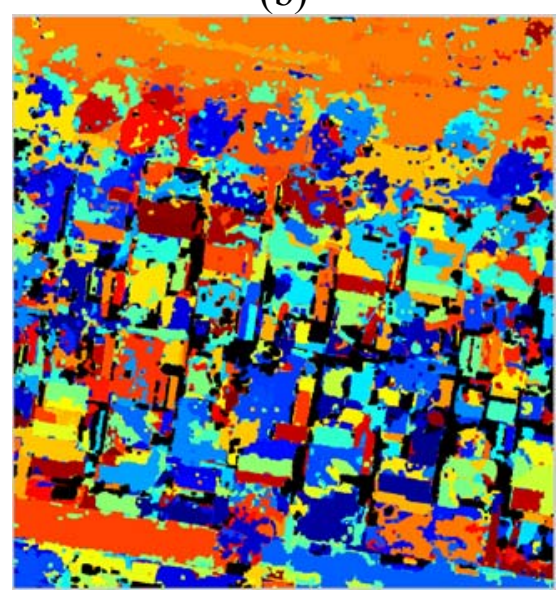

(c)

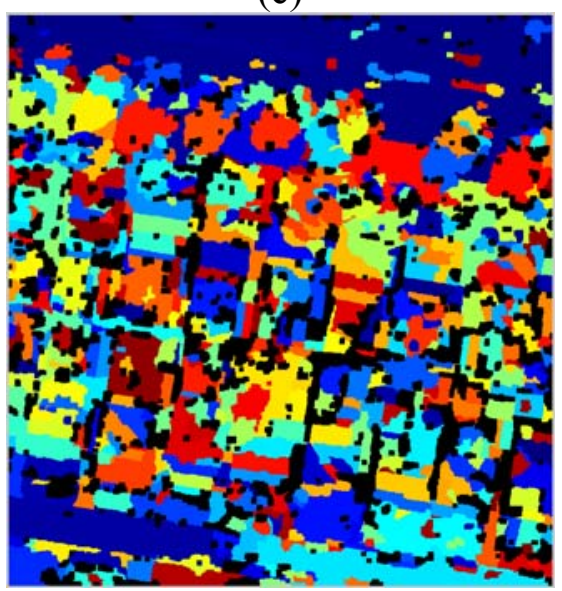

\subsection{Vegetation Identification}

The next phase of the algorithm is to identify and remove vegetation. Vegetation candidate pixels are identified by Otsu thresholding [12], a first-order color invariant [11]. Then, if $60 \%$ of a given region, identified via the aforementioned color segmentation algorithm, contains vegetation candidate pixels, then that entire region is labeled as a vegetation region. Color invariants, originally proposed by Gevers et al. in [11] are a set of color models independent of the viewpoint, surface orientation, illumination direction, illumination intensity, and highlights. Similar to Sirmacek et al. in [10], we extract the color information in the aerial image using the color index proposed by Unsalan and Boyer in [15]. The following color invariant is defined with the green and blue channels of the color aerial image:

$$
\psi_{g}(i, j)=\frac{4}{\pi} \cdot \arctan \left(\frac{I(i, j, g)-I(i, j, b)}{I(i, j, g)+I(i, j, b)}\right) \forall i, \forall j \quad i \in\{1, \ldots, R\}, j \in\{1, \ldots, C\}
$$


Sirmacek and Unsalan observed that green subtracted from blue primarily detected shadows. It was observed that blue from green in turn primarily identified vegetation. Any combination of using the red spectrum overlapped with identifying building roof tops and was avoided for vegetation detection.

The color invariant image (1) is then Otsu thresholded and all pixels having values in the image falling above that threshold $T_{g}$ are marked as vegetation candidates $V(i, j)$. This produces a binary image with white/true pixels corresponding to vegetation candidates and black/false pixels corresponding to non-vegetation candidates:

$$
\left[V(i, j)=1 \mid \psi_{g}(i, j)>T_{g}\right] \wedge\left[V(i, j)=0 \mid \psi_{g}(i, j)<=T_{g}\right] \forall i, \forall j \quad i \in\{1, \ldots, R\}, j \in\{1, \ldots, C\}
$$

This method will produce single, isolated pixels in places that do not actually correspond to vegetation. Therefore, the color invariant image is processed by a morphological closing operation (image dilation followed by erosion) followed by an opening operation (image erosion followed by dilation). Even after this processing, small groups of pixels will still sometimes exist where vegetation does not. However, these small groups of false positives do not compose the majority of the color segmented region in which they exist in. Let the variable $l_{v c}$ be the set of all vegetation candidates $\mathrm{V}(\mathrm{i}, \mathrm{j})=1$ (after morphological opening and closing operators) which exist in the color segmented region $l_{o}$ :

$$
l_{v c}=\left\{V(i, j) \mid\left[O(i, j)=l_{o}\right] \wedge[V(i, j)=1]\right\}
$$

Then, the total number of vegetation candidate pixels belonging to region $l_{o}$ would be $S\left(l_{v c}\right)$ and the percentage of vegetation candidate pixels occupying a given region would be $S\left(l_{v c}\right) / S\left(l_{o}\right)$. We therefore implement the rule that a given region $l_{o}$ and correspondingly all its member pixels are classified as a vegetation region $l_{v}$ provided that at least $60 \%$ of the pixels in the region $l_{o}$ are classified as vegetation candidates. Let the set of all vegetation regions be denoted as $L_{v}$ where $1_{\mathrm{v}} \in \mathrm{L}_{\mathrm{v}}$ :

$$
L_{v}=\left\{l_{o} \rightarrow l_{v} \mid \frac{S\left(l_{v c}\right)}{S\left(l_{o}\right)}>0.6\right\}
$$

The steps of the vegetation detection algorithm are depicted in Figure 2.

Figure 2. Vegetation Detection Block Diagram.

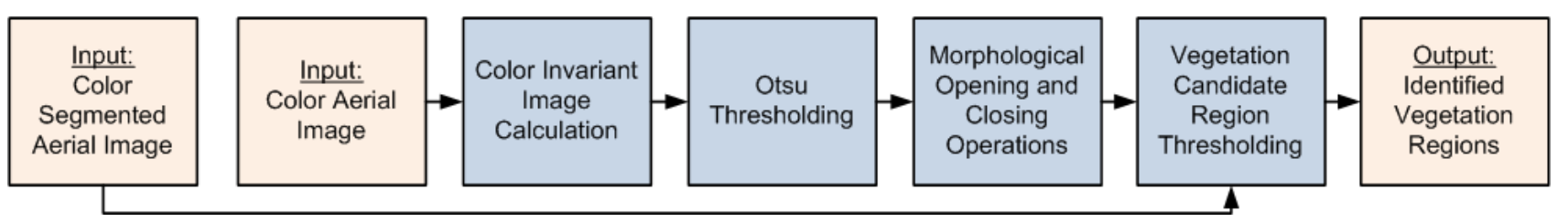

Plots at several stages of the vegetation detection algorithm are shown in Figure 3.

While the majority of the vegetation in Figure 3 is correctly labeled as vegetation, some other portions of the image are incorrectly labeled as vegetation. If non-vegetation pixels share similar spectral attributes with vegetation (such as certain hues of green) it is possible that if enough of those pixels are clumped together, they will be erroneously labeled as vegetation. 
Figure 3. Output images at various stages of the vegetation detection algorithm. (a) Shows the original aerial input image; (b) shows the final color segmented version of that image; (c) shows the output after Otsu thresholding the green color invariant where white pixels are marked as vegetation candidates; (d) shows the final vegetation regions after morphological opening and closing and thresholding the regions for sufficient amount of vegetation candidates.

(a)

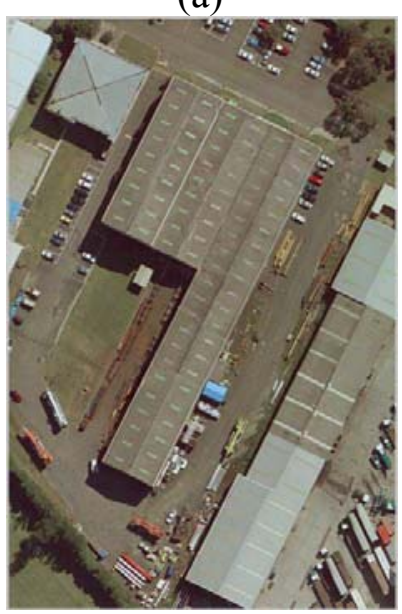

(b)

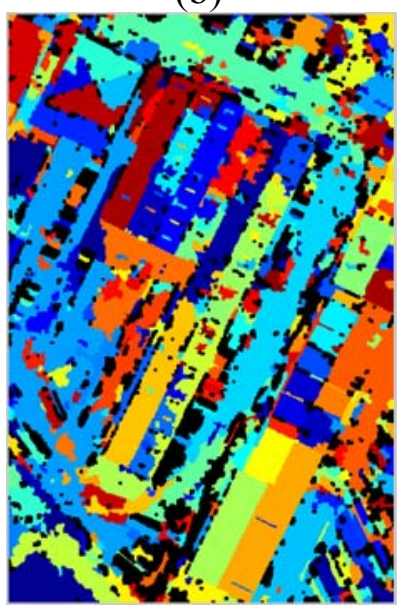

(c)

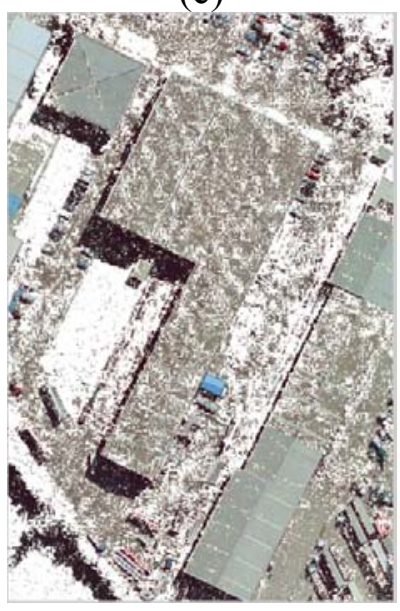

(d)

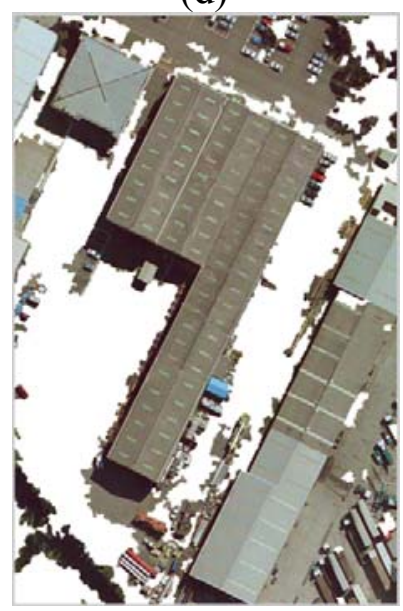

\subsection{Shadow Region Identification}

Shadows are identified through the use of a modified color invariant. Sirmacek et al. in [10] used an equation similar to (1) except they took the difference between the blue and green color channels over the sum of those channels to identify shadows. We have found better success with instead using the image constructed by the following equation:

$$
\psi_{s}(i, j)=\frac{4}{\pi} \cdot \arctan \left(\frac{I(i, j, r)-\sqrt{I(i, j, r)^{2}+I(i, j, g)^{2}+I(i, j, b)^{2}}}{I(i, j, r)+\sqrt{I(i, j, r)^{2}+I(i, j, g)^{2}+I(i, j, b)^{2}}}\right) \quad \forall i, \forall j \quad i \in\{1, \ldots, R\}, j \in\{1, \ldots, C\}
$$

This image is then Otsu thresholded and all pixels below the threshold are considered shadow. In Figure 4, Sirmacek's equation is implemented on the middle image (using the difference between the blue and green channels over their sum) and our method from equation (5) is shown on the right where pixels appearing completely white are shadow pixels. In our experience, it is unlikely that a building casts a shadow on another building. Notice that Sirmacek's method winds up labeling pixels on the dark side of the roof as shadows and our approach does not. This is important to our methodology as we will end up removing all pixels identified as shadow by this technique and ultimately considering shadow and building as mutually exclusive classes. 
Figure 4. Shadow detection result comparison. (a) Original aerial image; (b) Sirmacek and Unsalan's method; (c) our method.

(a)

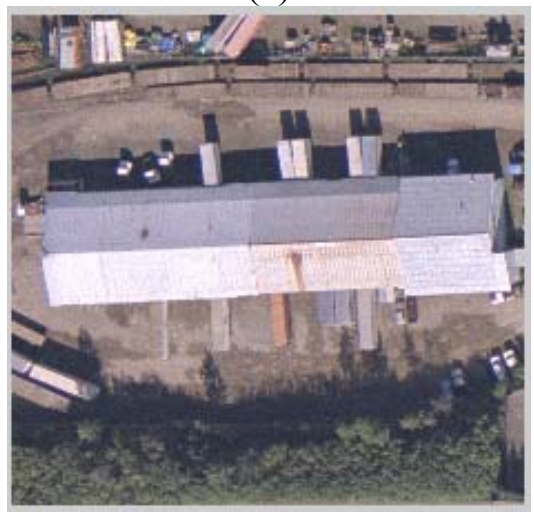

(b)

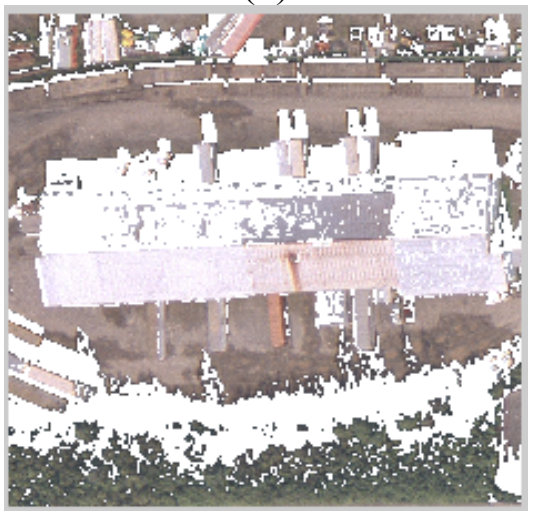

(c)

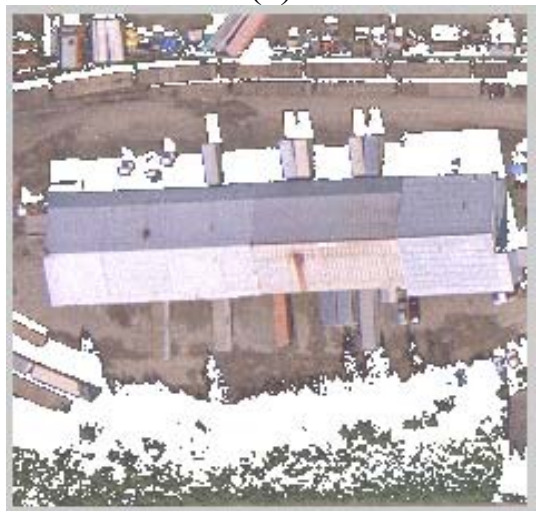

\subsection{Building/Non-Building Identification and Entropy Segmentation}

The color segmenting technique in Section 2.1 sometimes segments the image with too many segments, but the borders of those segments are fairly accurate in outlining the objects they approximate in the image. An entropy segmenting technique is used for building identification that has a parameter to control the coarseness of the generated segments. Buildings and non-buildings (other than shadow and vegetation) are detected by measuring the solidity of their regions. The regions are obtained by entropy filtering the input image and then using a watershed segmentation technique on that entropy filtered image.

We used Matlab's entropyfilt function [16] to produce an entropy image. The entropy filter (see Chapter 11 of 17) produces an entropy image where each pixel is the entropy calculated from a $9 \times 9$ neighborhood in the input image I. Entropy is calculated as follows:

$$
e=-\sum_{i=1}^{L} p\left(z_{i}\right) \cdot \log _{2}\left(p\left(z_{i}\right)\right)
$$

In equation (6), $p\left(z_{i}\right)$ is the number of occurrences the intensity $z_{i}$ has in the $9 \times 9$ neighborhood. The entropy image $I$ is subsequently thresholded (we removed all pixels less than 0.75 times the maximum entropy value in the entropy image) and then a watershed segmentation 18 is applied to the distance transform of the thresholded entropy image. For our purposes, the 0.75 worked well for the coarse segmentations we desired. The entropy segmentation algorithm block diagram depicted in Figure 5 .

Figure 5. Entropy Filtering / Watershed Entropy Segmentation Algorithm Block Diagram.

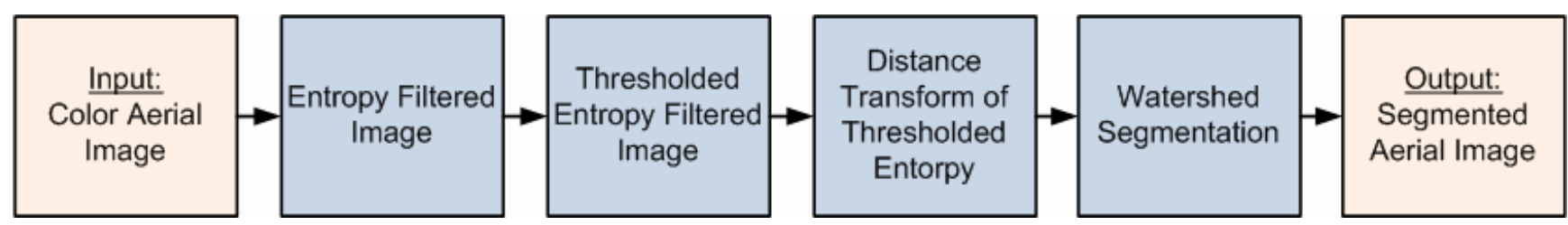


Figure 6. The subfigures show the outputs of the entropy/watershed entropy segmentation technique at various stages. (a) Entropy image; (b) The thresholded entropy image; (c) The distance transform of the thresholded entropy image; (d) The watershed segmentation applied to output in (c).

(a)

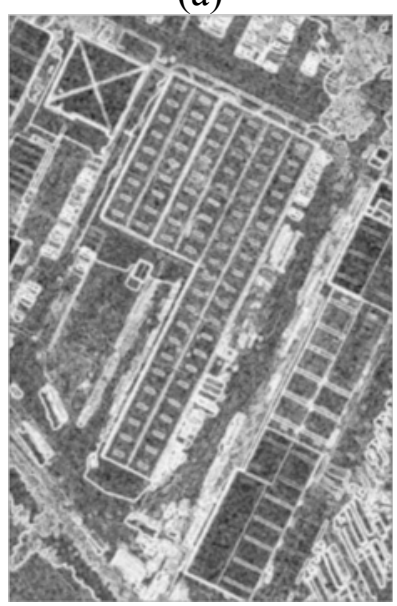

(b)

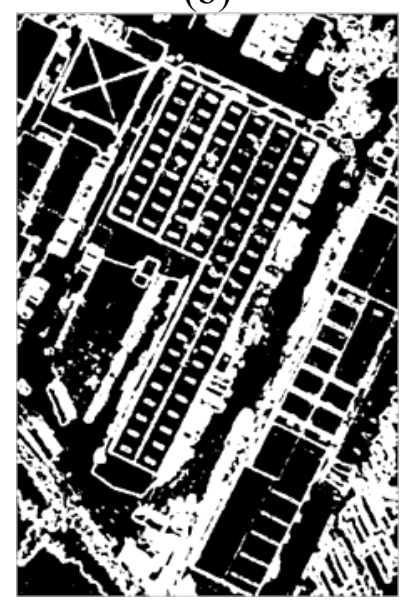

(c)

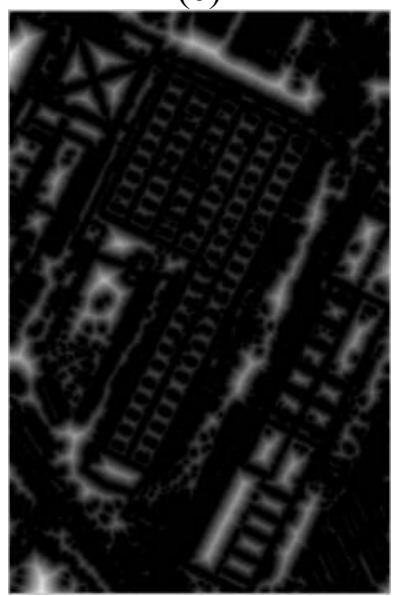

(d)

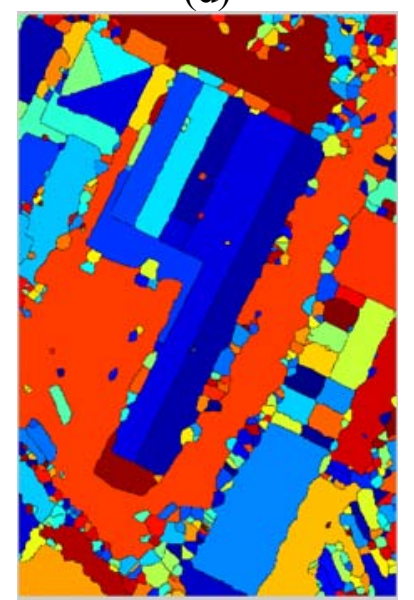

A convex hull is defined here as the minimum polygon which completely encompasses a given region and is calculated with Matlab's regionprops command 19. Consider the black region in Figure 7 (a), its area is simply the sum of all the black pixels. The convex hull for that region is then shown in Figure 7 (b) in yellow and the area for that convex hull is in Figure 7 (c) (the sum of all the yellow pixels).

Figure 7. Convex hull example. (a) Arbitrary region; (b)Yellow convex hull which bounds the exterior of the arbitrary region; (c) Area of that convex hull.

(a)

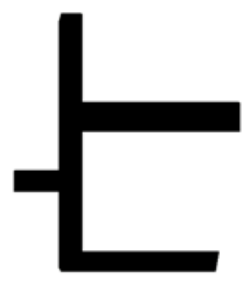

(b)

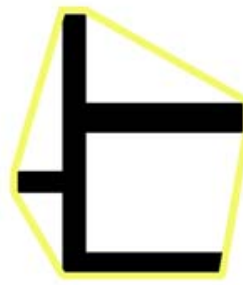

(c)

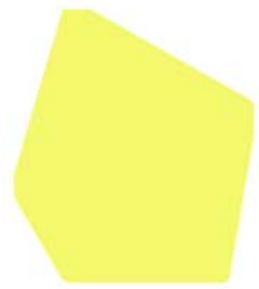

Let a region created by the watershed segmentation technique be denoted as $l_{w}$ where $L_{w}$ is the set of all regions created and $1_{\mathrm{w}} \in \mathrm{L}_{\mathrm{w}}$. Let the set of all pixels which composes the convex hull (filled in area as shown in Figure 7 (c)) which bounds $l_{w}$ be denoted as $c_{w}$ and the size of that convex hull be denoted as $S\left(C_{w}\right)$ (note this size is defined as the area within the convex hull or sum of all yellow pixels in Figure 7 (c)). Let the size of the watershed segmentation region be denoted as $\mathrm{S}\left(1_{\mathrm{w}}\right)$. Let the solidity for the watershed segmentation region $1_{w}$ be denoted as $D_{w}$. Solidity is then defined as follows:

$$
D_{w}=\frac{S\left(l_{w}\right)}{S\left(c_{w}\right)} \quad D_{w} \in(0,1]
$$


The upper limit of the solidity can be exactly one (the region itself is a convex hull) and the lower limit can get infinitely close to 0 . Therefore, the more convex the region exterior is, the higher its solidity. Notice that for even in the largest building in the center of Figure 6, most of the building is broken into regions having a high solidity. However, the largest segment surrounding the center building in Figure 6 (appearing in orange) is not approximated very well by a convex hull and has a low solidity. We have noticed roads and surrounding landscapes are typically approximated by a single region with this entropy segmentation approach and often have low solidity values. We therefore label all regions having a solidity above 0.7 as building and everything equal to or below as non-building. This approach implements the assumption that the roof sections of a building can be approximated with convex hull regions. Note that this does not necessarily assume the entire building roof has to have a high solidity, just the various sections composing its roof. However, if a single building exists as an "L" shape and has a single section across its entire roof, then this approach would most likely erroneously classify such a building as non-building. If roads are broken up into small, convex sections, then they unfortunately will be mistaken as buildings.

A block diagram showing all of the aforementioned components of the proposed vegetation identification and building detection system are shown in Figure 8. After the region solidity calculation identifies building candidate regions, any pixels which belong to building candidate regions that were also marked as either vegetation or shadow are then disqualified as building candidate pixels.

Figure 8. Building Detection and Image Classification Block Diagram.

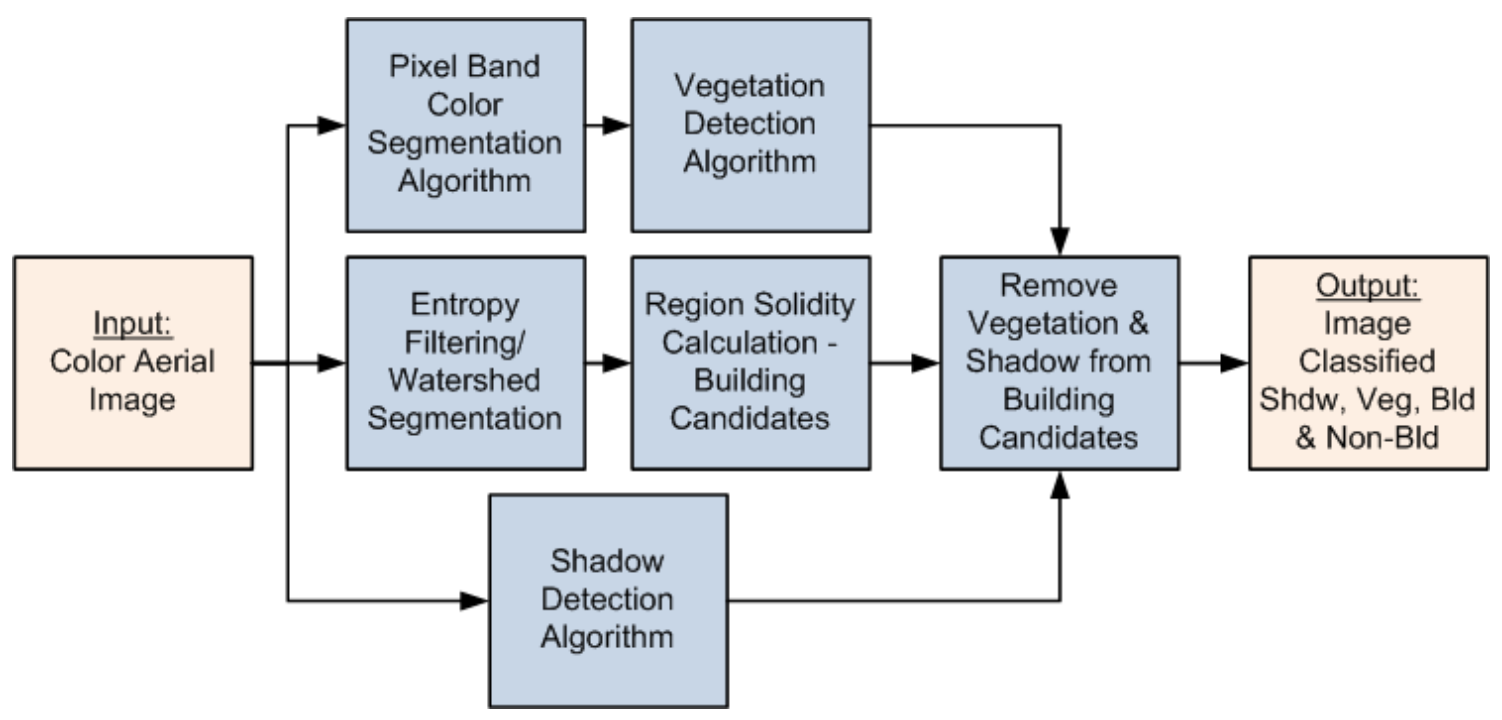

\section{Experimental Results}

\subsection{Empirical Parameters}

The entropy image threshold (0.75), the color segmentation bin quantization (17), the region solidity (0.7) and the vegetation region threshold (0.6) were empirically determined by qualitatively examining the visually best results demonstrated on a small subset of the Fairfield data set (five images totaling in size to $7 \%$ of the actual dataset). Note, in evaluating the parameter selection, those images 
were visually examined; the reference set was never consulted during this phase nor were parameter sweeps done. These parameters were then held fixed for the algorithm's execution across both the Fairfield and Anchorage data sets.

\subsection{Data Sets}

The proposed algorithm has been tested on two different data sets. The first data set depicts a $2 \times 2 \mathrm{~km}^{2}$ area from Fairfield (Australia) and is referred to from here on out as "the Fairfield data set". This data set includes LiDAR data, containing first and last return pulse information, as well as returned pulse intensity. The data set also has a RGB aerial color image, which is depicted in Figure 9, having $15 \mathrm{~cm}$ pixel resolution. A suburban area exists to the southwest whereas the northeast mostly contains industrial buildings.

Figure 9. Fairfield Data Set Aerial Imagery.

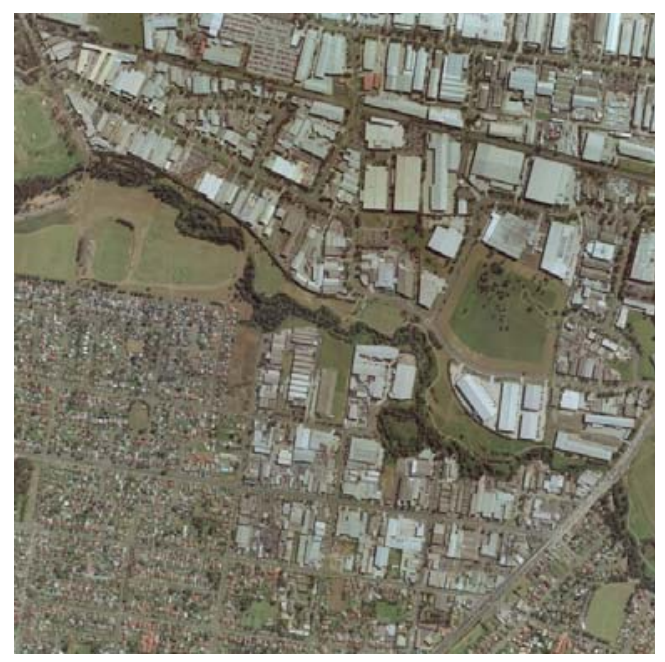

Figure 10. Anchorage Data Set Aerial Imagery.

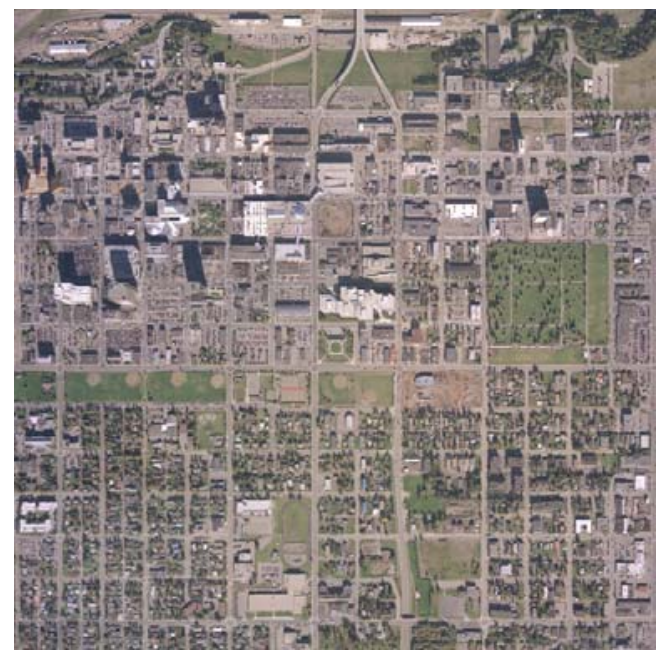


A cropped portion of Figure 9, showing the residential buildings existent in the south west portion of the data set, is shown in Figure 11. The manual identification of the buildings in Figure 11 is shown in Figure 12.

Figure 11. Cropped Residential Portion of the Fairfield Data Set Aerial Imagery.

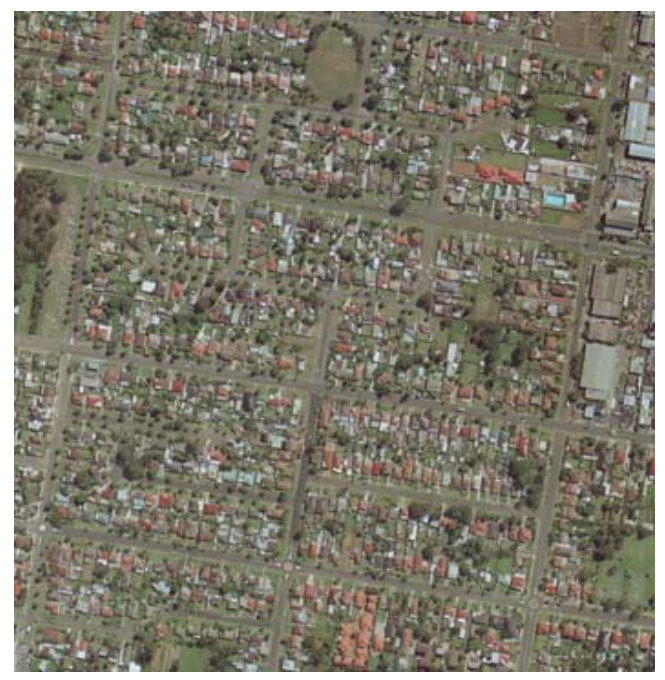

Figure 12. Cropped Residential Portion of the Manually Identified Buildings in Fairfield Data Set.

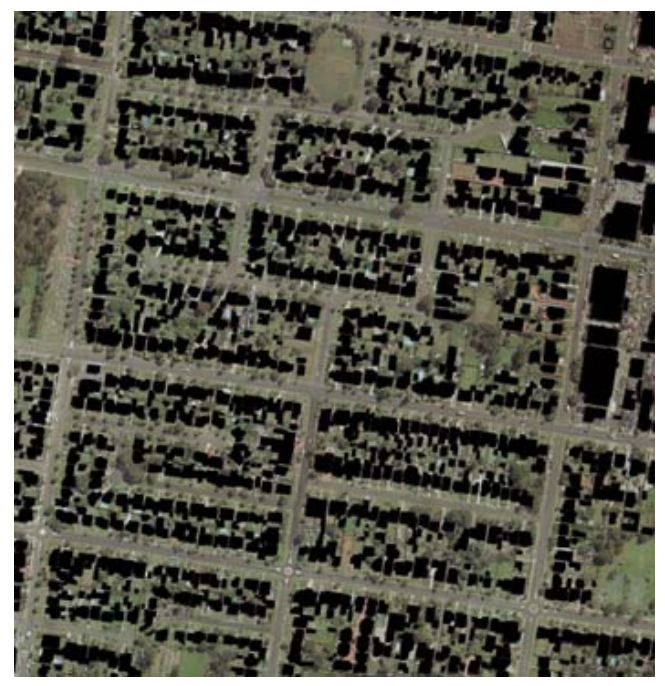

The second data set depicts a $1.67 \times 1.67 \mathrm{~km}^{2}$ area from Anchorage (Alaska, USA) and from here on out is referred to as "the Anchorage data set". This data set includes both LiDAR data, with first return pulses and no last return nor returned intensity, and aerial imagery, shown in Figure 10, existing as an RGB color image at $15 \mathrm{~cm}$ pixel resolution with a planned horizontal accuracy of $60 \mathrm{~cm}$.

In both data sets, the LiDAR data and the aerial image depict the terrain from a nadir/top down perspective and the accompanying aerial image has been ortho rectified. The Fairfield data set has different building and terrain characteristics when compared to the Anchorage data set. The industrial portion of the Fairfield data set is not by any means equivalent to the commercial portion of the Anchorage data set. The buildings in the industrial portion of the Fairfield data set, for the most part, 
lie relatively low and have a large base, whereas some of the commercial buildings in the Anchorage data set rise fairly high. Because the sun is lower to the ground when the Anchorage data set was captured, the shadows in the Anchorage are longer than the Fairfield data set. Furthermore, the Anchorage data set to the North East contains a highly populated urban area filled with a great deal of concrete and little vegetation. There is some dense forestry to the North West of the Anchorage data set. Whereas there exists more vegetation in the industrial part of the Fairfield Data Set to the North West (in comparison to the Anchorage data set's commercial sector) and there's a dense forestry spanning through the center of the data set.

In order to evaluate the accuracy of the proposed algorithm, building pixels were first manually identified in both data sets. The Fairfield data set contained a total of 1,401 building regions and the Anchorage Data set contained 1,242 building regions. This set of manually labeled points will be referred to as the reference set. The proposed building detection algorithm labels all points belonging to different buildings with unique building labels. From here on out this will be referred to as the automatic set.

\subsection{Evaluation Metrics}

For evaluating the automatically extracted buildings, using a reference data set, two performance metrics are pertinent - the completeness and the correctness of the results [20]. On a pixel level, the completeness represents the percentage of pixels belonging to buildings that were correctly detected by the algorithm. The correctness represents the percentage of pixels labeled by the algorithm as building that actually correspond to building points.

On a building level, the completeness represents the percentage of buildings that are correctly detected by the algorithm and the correctness represents the percentage of buildings that the algorithm identified that are actually buildings which exist in the reference set. The building level completeness and correctness metrics used in this research are calculated the same way in which they are defined by Rottensteiner et al. in Section 3.2 of [2] which were also used in [3].

\subsection{Building Detection Results: Pixel and Building Level Completeness and Correctness}

The pixel level completeness and pixel level correctness achieved by the proposed building detection algorithm for the Fairfield data set are 0.8258 and 0.6163 , respectively. This means that $82.6 \%$ of the pixels belonging to buildings, according to the reference set, were correctly detected by the algorithm in the automatic set and that $61.6 \%$ of the pixels labeled as building by the algorithm in the automatic set were actually also labeled building in the reference set. The pixel level completeness and pixel level correctness for the Anchorage data set are 0.7486 and 0.4154, respectively. Again, $74.9 \%$ of the pixels belong to buildings were correctly detected by the algorithm and $41.5 \%$ of the pixels labeled as building by the algorithm actually corresponded to building pixels. Confusion matrices for the results from both data sets are shown in Table 1 and Table 2. The algorithm realized a kappa value of 0.5613 on the Fairfield Data set and 0.34109 for the Anchorage Data set. 
Table 1. Confusion Matrix for Fairfield Data Set.

\begin{tabular}{|c|l|l|r|}
\hline \multicolumn{2}{|c|}{} & \multicolumn{2}{c|}{ Auto } \\
\cline { 3 - 5 } & Bld & Non-Bld \\
\hline \multirow{4}{*}{$\approx$} & Bld & $42,279,727$ & $8,920,741$ \\
\cline { 2 - 4 } & Non-Bld & $26,321,752$ & $100,433,380$ \\
\hline
\end{tabular}

Table 2. Confusion Matrix for Anchorage Data Set.

\begin{tabular}{|c|l|l|c|}
\hline \multicolumn{2}{|c|}{} & \multicolumn{2}{c|}{ Auto } \\
\cline { 3 - 5 } & Bld & Non-Bld \\
\hline \multirow{2}{*}{$\approx$} & Bld & $20,260,940$ & $6,803,900$ \\
\cline { 2 - 4 } & Non-Bld & $28,515,278$ & $63,142,698$ \\
\hline
\end{tabular}

Figure 13 and Figure 14 presents pixel level results for the entire Anchorage and Fairfield data sets respectively. In these figures, the blue color corresponds to correctly detected points, the light green color corresponds to false positives (points the algorithm classified as belonging to buildings in the automatic set but were in fact identified as not belonging to buildings in the reference set) and the red color corresponds to false negatives (points that were identified as belonging to buildings in the reference set but classified as not belonging buildings in the automatic set by the algorithm). The white in these figures corresponds to points not identified as building by both the algorithm in the automatic set and the manual extraction in the reference set (true negatives).

Observe that in Figure 13 and Figure 14 almost all of the false positive pixels correspond to either roads, sidewalks, parking lots, or dirt. Some of the roads have been correctly classified as non-building because their solidity is too low when represented by a single segment. The majority of the false negatives are smaller buildings or are due to buildings casting shadows on other buildings. The algorithm performs very well in not mistaking vegetation for building.

Figure 15 and Figure 16 present pixel classification results as buildings, non-buildings, shadow and vegetation for entire the Anchorage and Fairfield data sets respectively. The red pixels correspond to what the proposed algorithm classified as building, the green pixels as vegetation, the blue pixels as shadow and the white pixels as non-building. Unfortunately, the darker side of trees is often classified as shadow, which is correct however the trees typically overcast vegetation. Most of the non-building correctly corresponds to concrete surfaces such as roads or parking lots, which are not parts of buildings. Again, observe that the algorithm performs very well in identifying the sun side part of the dense forestry and the rest of the vegetation existent in the data set. 
Figure 13. Building Pixel Accuracy for the Anchorage Data Set.

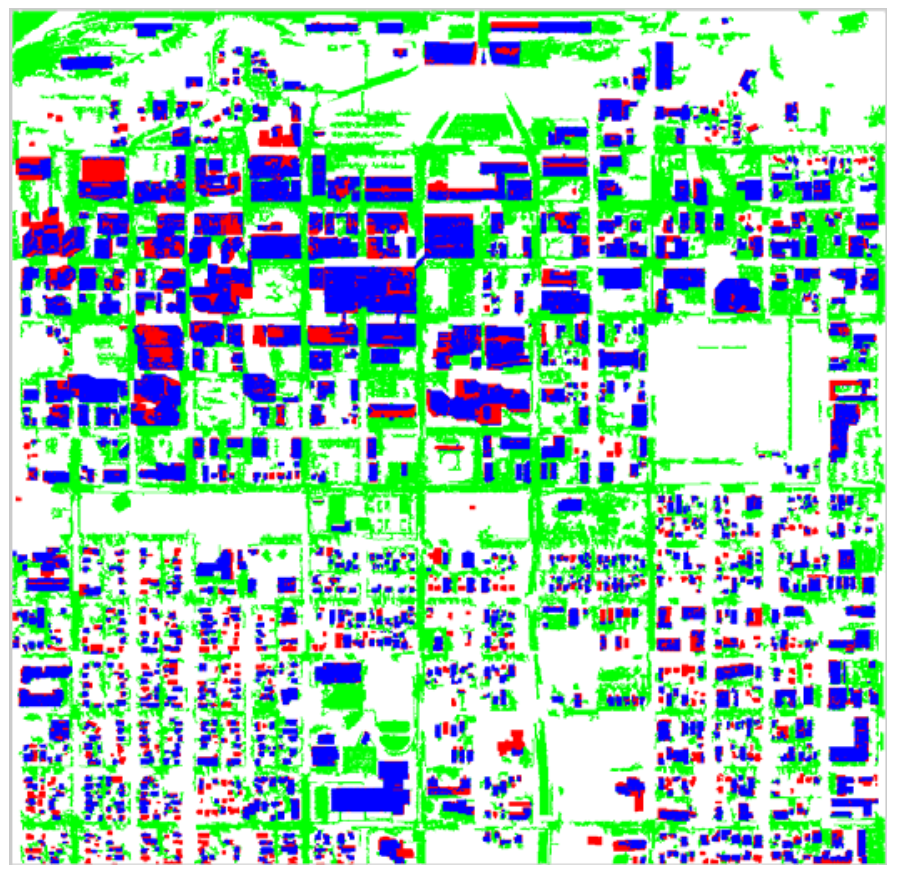

Figure 14. Building Pixel Accuracy for the Fairfield Data Set.

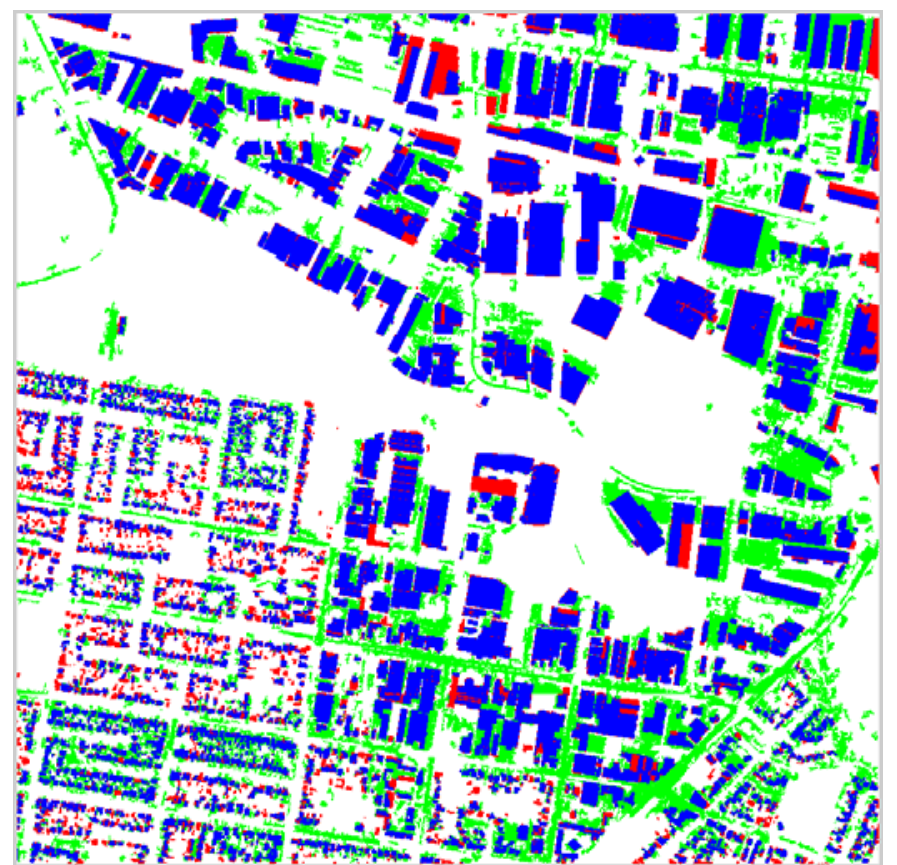


Figure 15. Pixel Classification for Anchorage Data Set.

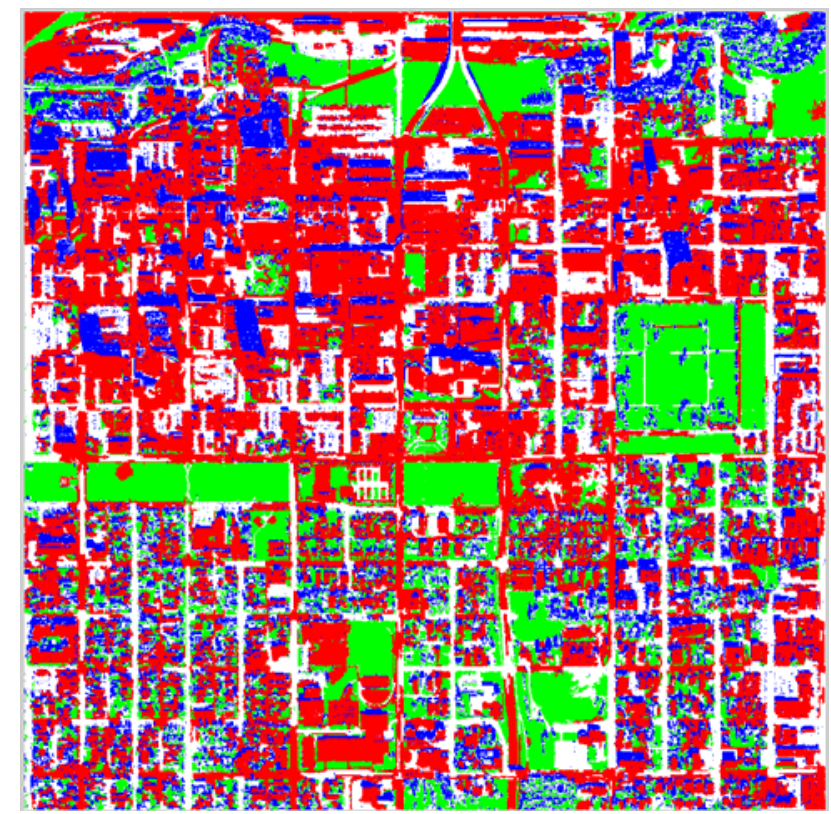

Figure 16. Pixel Classification for Fairfield Data Set.

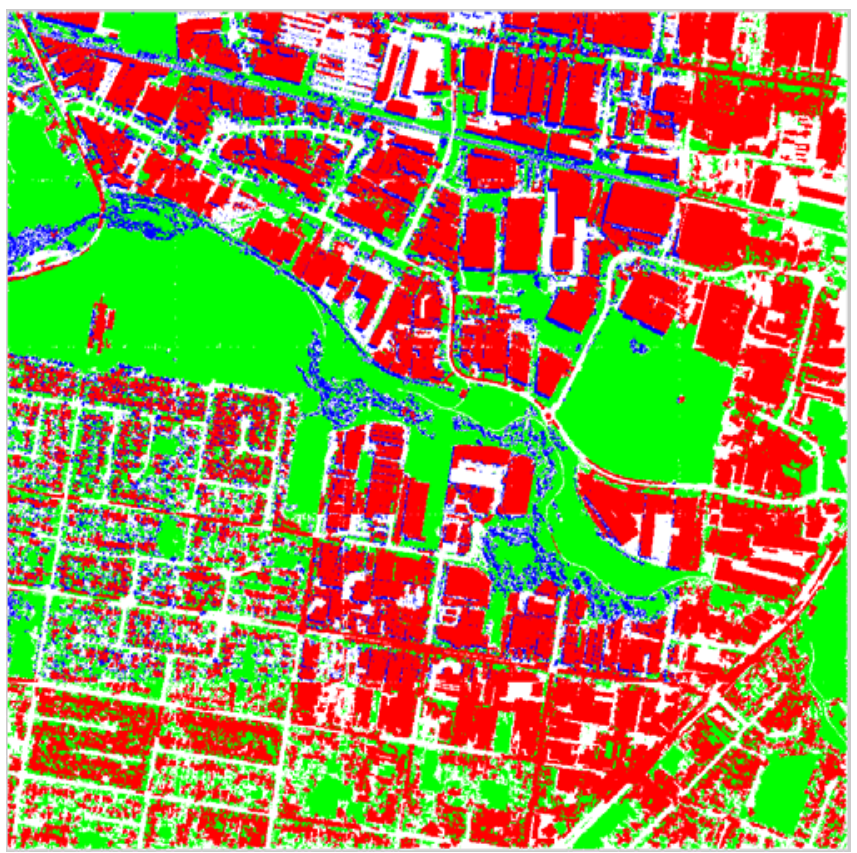

Figure 17 and Figure 18 present pixel level results for a cropped residential portion of the Fairfield data set. 
Figure 17. Cropped Residential Portion of the Building Pixel Accuracy for the Fairfield Data Set.

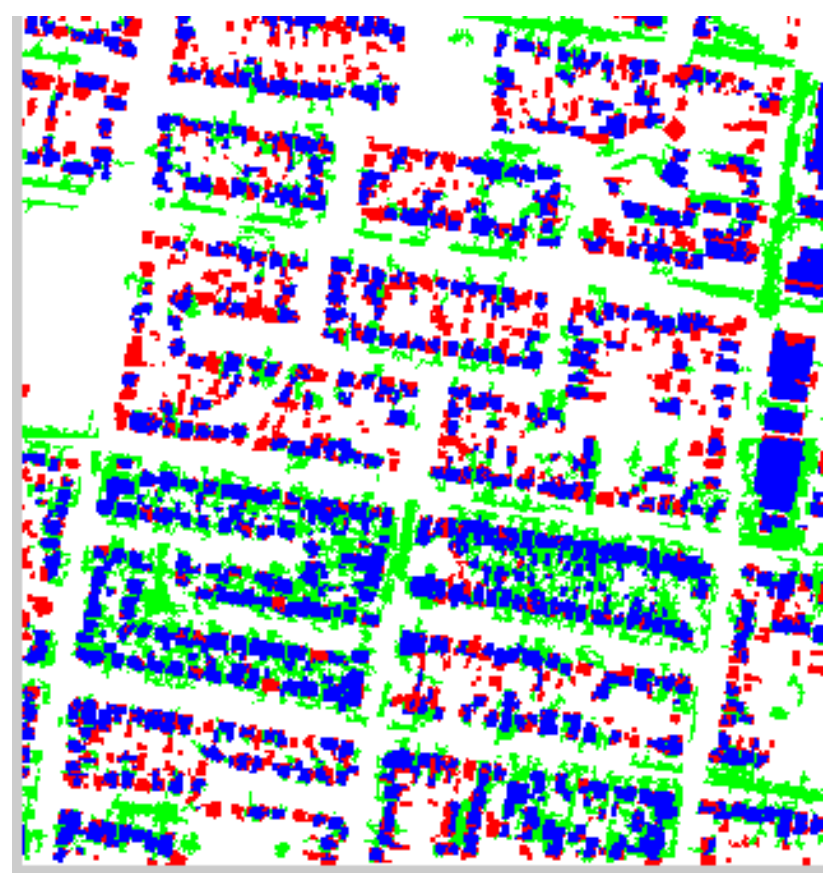

Figure 18. Cropped Residential Portion of the Pixel Classification for Fairfield Data Set.

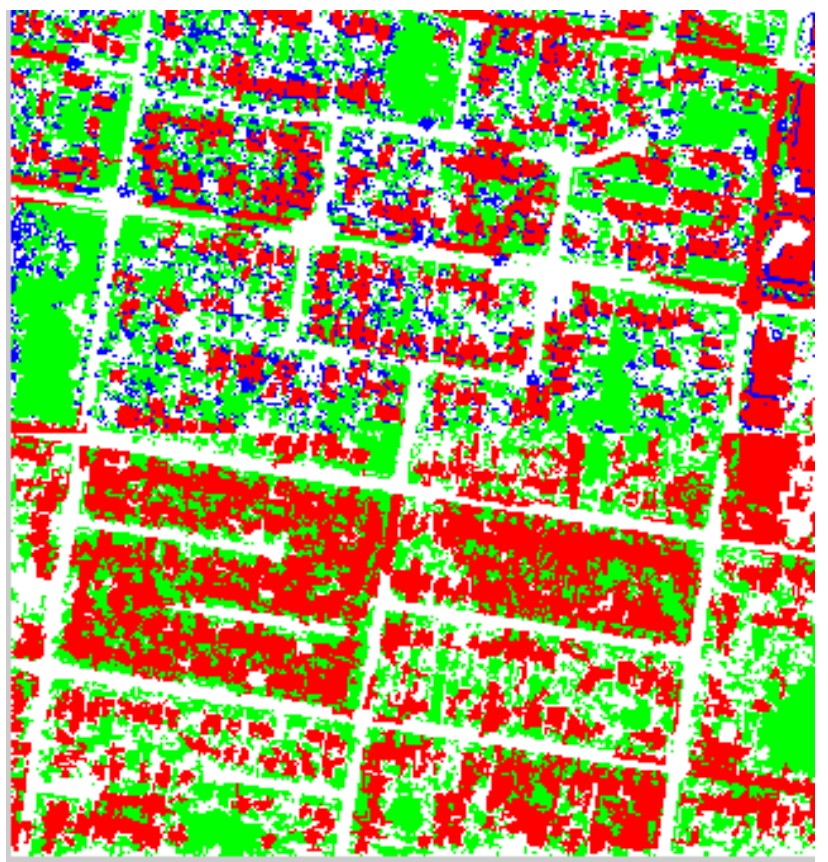

The building level completeness and correctness results, as a function of various building areas, for the Anchorage data set are shown in Figure 19. Figure 20 presents cumulative completeness and correctness which is simply the algorithm's completeness and correctness for all buildings having an area greater than the value shown on the $\mathrm{x}$-axis. 
Figure 19. Completeness and Correctness for the Anchorage Data Set.

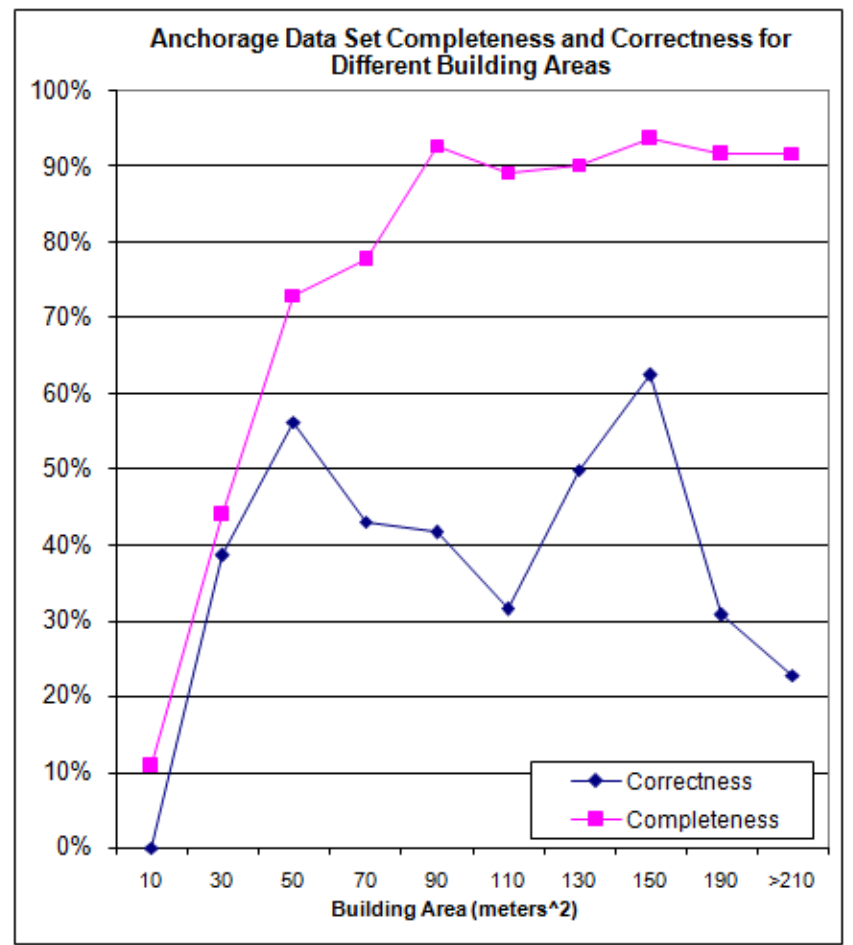

Figure 20. Cumulative Completeness and Correctness for the Anchorage Data Set.

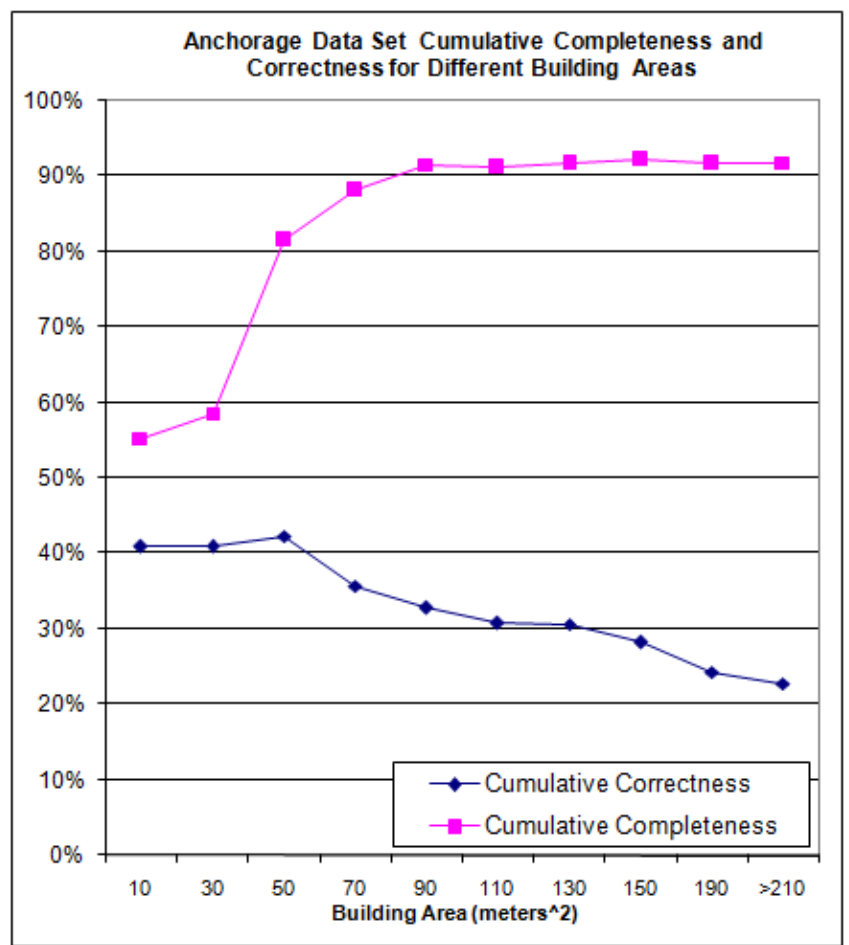

The building level completeness and correctness for the Fairfield data set is shown in Figure 21 and the cumulative completeness and correctness in Figure 22. 
Figure 21. Completeness and Correctness for the Fairfield Data Set.

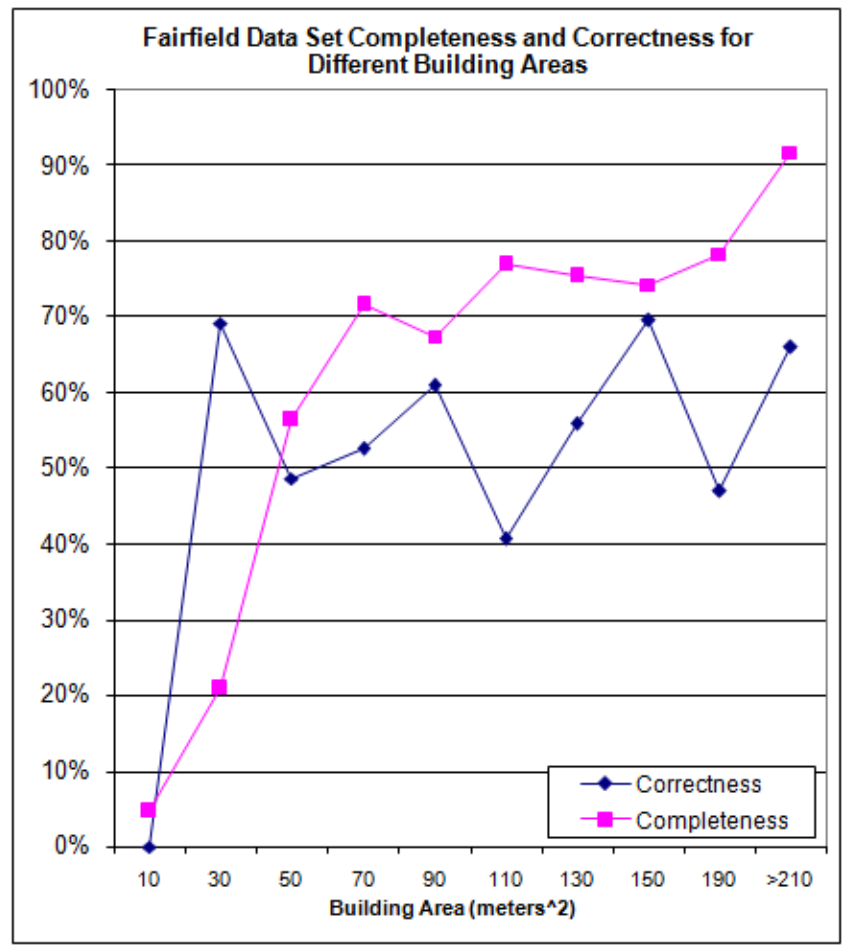

Figure 22. Cumulative Completeness and Correctness for the Fairfield Data Set.

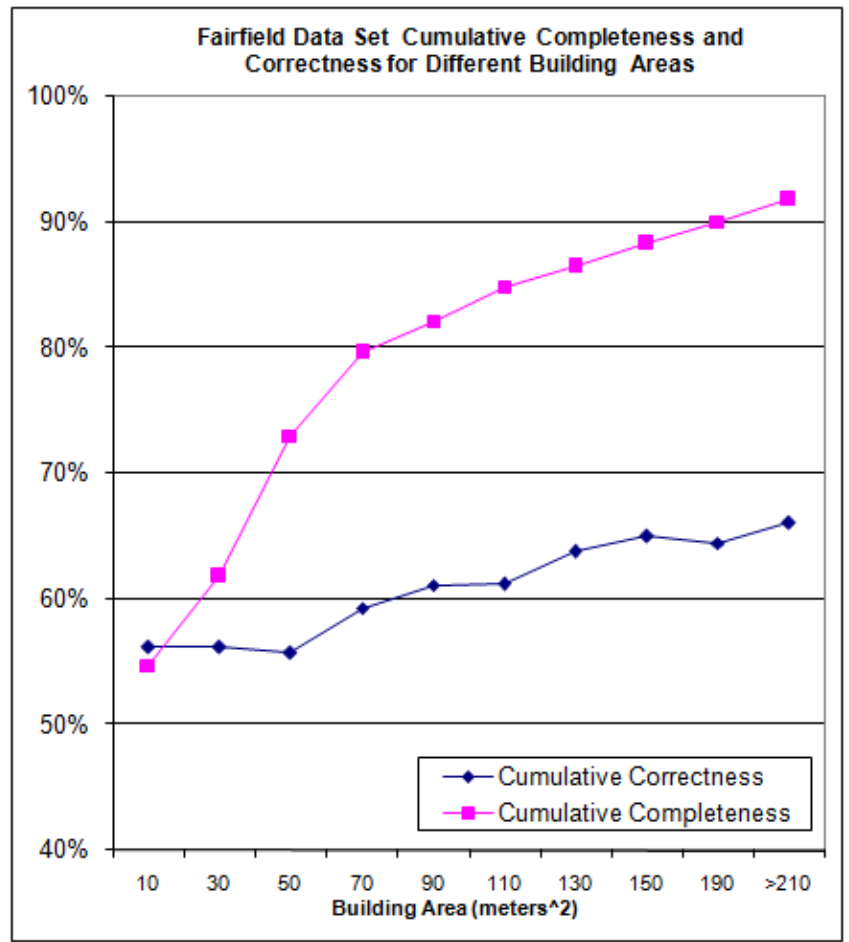

In Figure 22 note that for a building area of $150 \mathrm{~m}^{2}$ for the Fairfield data set, the algorithm has a cumulative completeness and cumulative correctness of approximately $88 \%$ and $65 \%$, respectively. This simply means that the algorithm's completeness and correctness for all buildings having an area of $150 \mathrm{~m}^{2}$ and greater is $88 \%$ and $65 \%$ (respectively). The range was plotted from between $10 \mathrm{~m}^{2}$ 
to $210 \mathrm{~m}^{2}$ as after $210 \mathrm{~m}^{2}$. The algorithm detects $80 \%$ of the buildings larger than $70 \mathrm{~m}^{2}$ but at the same time its correctness suffers at the expense of false positives. The reason for the mediocre correctness is the algorithm is completely automated: no parameters change during the execution across the data sets. Also, no assumptions are implemented to tailor to a specific building size or shape. As it is shown soon via the data set building histogram, there are a variety of different building sizes in the data set. Finally, the mediocre correctness is also because the automatic algorithm is detecting these buildings from only a single nadir aerial image.

The histogram for building sizes the Anchorage data set is shown in Figure 23 and for the Fairfield data set in Figure 24. The height of the red and yellow bars represent the total number of buildings manually extracted for the building area listed on the $\mathrm{x}$-axis. The height of the blue and gray bars represent the total number of buildings identified by the algorithm for the building area listed on the $\mathrm{x}$-axis. The red part of the red and yellow bar is proportional to the amount of those buildings which were detected by the algorithm whereas the yellow portion of the bar represents the buildings the algorithm failed to detect for the given area on the $\mathrm{x}$-axis. The blue portion of the blue and gray bars represents the number of buildings the algorithm detected which actually corresponded to buildings in the reference set and the gray portion represents the number of buildings the algorithm claimed to be building but did not correspond to any buildings identified in the reference set for the building area listed on the $\mathrm{x}$-axis.

Figure 23. Anchorage Data Set Histogram.

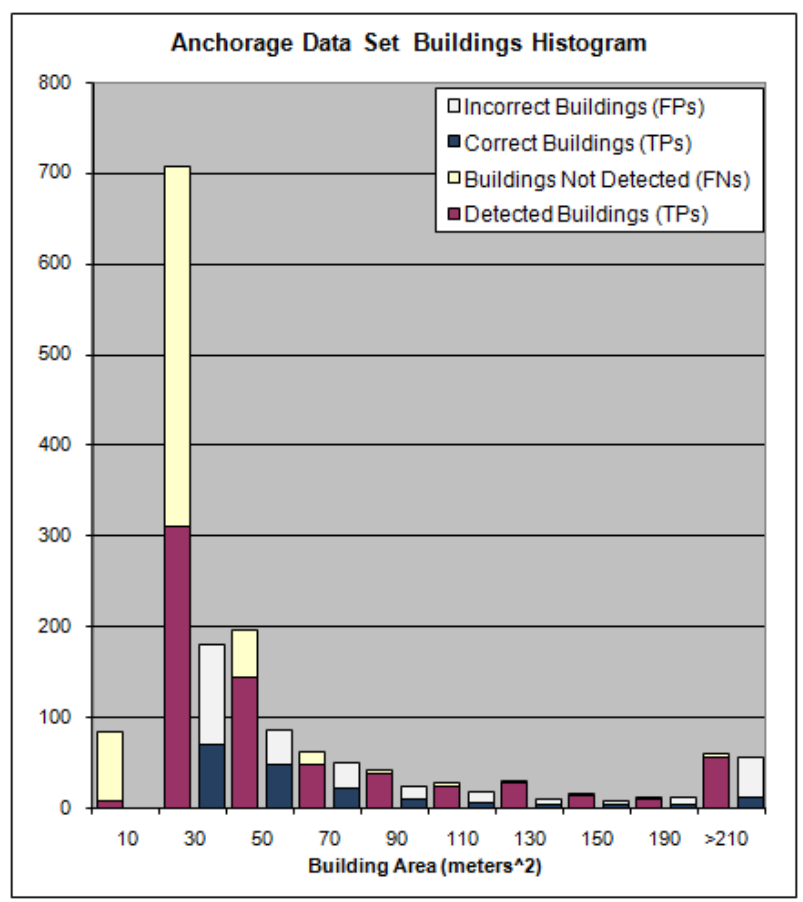


Figure 24. Fairfield Data Set Histogram.

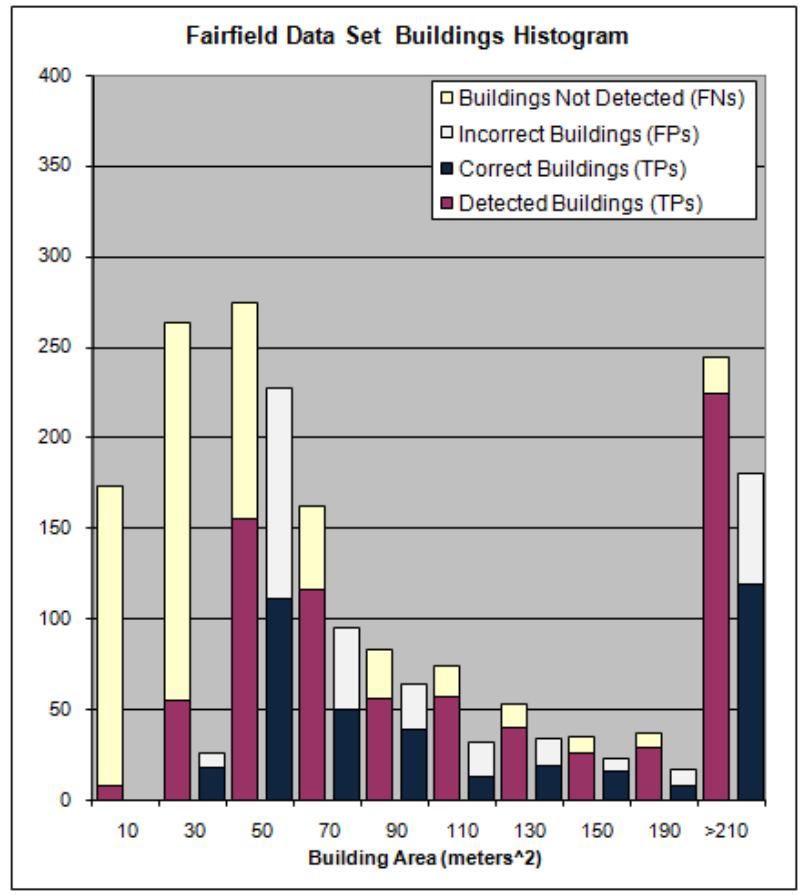

\subsection{Vegetation Detection Results: Pixel Correctness and Percent Coverage}

We mentioned earlier that for the Fairfield data set there is also LiDAR data because it is possible to use the returned LiDAR intensity and one of the channels from the aerial image to compute a pseudo normalized difference vegetation index (NDVI) and threshold that NDVI image to identify vegetation. Rottensteiner et al. in [3] propose using the pseudo NDVI image calculate it by taking the difference of the returned LiDAR intensity at pixel $(i, j)$ and the red color component for that same pixel (from the RGB image) and divide that quantity by the sum of the returned LiDAR intensity and the red color component of the pixel. Note that in order to use the NDVI image, the LiDAR data must be interpolated and registered to the aerial imagery so that the LiDAR intensity at pixel $(i, j)$ and the red color component at $(\mathrm{i}, \mathrm{j})$ correspond to the same location in the depicted scene.

With the manually developed reference set, we know which pixels belong to buildings and which do not. We then tried 40 different thresholds (for thresholding the pseudo NDVI image to determine where vegetation exists). The thresholds ranged from -0.5 to 0.5 in increments of 0.025 . We then calculated the pseudo correctness for the red, blue and green channel based NDVI images for all the thresholds and plotted the accuracy. The pseudo correctness is calculated as follows:

$$
p C o r r=\frac{p T P}{p T P+F P}
$$

In equation (8) pTP is Pseudo True positive and FP is a false positive. If the thresholded NDVI labels a pixel as vegetation and that corresponding pixel is not labeled as belonging to a building in the reference set, then the pixel is classified as a pseudo true positive. The reason this is referred to as a 'pseudo' true positive' is because what is not building could be sidewalk, cement, etc. in addition to 
vegetation. A reference set was only developed for identifying buildings, not vegetation. If the thresholded NDVI labels a pixel as vegetation and that corresponding pixel is also labeled as belonging to a building in the reference set, then the pixel is classified as a false positive.

Because a reference set was not developed which identified the vegetation as ground truth, false negatives cannot be calculated. False negative pixels are pixels in which the thresholded NDVI classifies as not belonging to vegetation when in fact they do belong to vegetation. Instead only false positives can accurately be determined. Now, although in [3] Rottensteiner use the red channel, in earlier works [21], they report using the green channel of the aerial imagery. Figure 25 shows the pseudo correctness plotted for the red, green and blue thresholded NDVI images for all thresholds tested. In Figure 25, the red line corresponds to the NDVI constructed from the returned LiDAR intensity and the red channel of the corresponding color aerial imagery (similarly for the green and blue channels for the green and blue lines). Figure 25 shows the percentage of pixels classified as vegetation by the pseudo NDVI approach.

We tested our proposed color invariant vegetation detection approach on the Fairfield Data Set and the Anchorage Data Set and it realized a pseudo correctness of $97.25 \%$ and $96.91 \%$, respectively. Note that our approach only requires the aerial imagery and is automatic - no parameter adjustment. A comparison of the best NDVI results and ours are presented in Table 3 where our method is listed as 'Clr Inv Veg' and the best results from thresholding the different channel NDVI images are listed as 'NDVI Red', 'NDVI Blue' and 'NDVI Green'. Not only does our approach realize a significantly higher correctness, but it is also labeling more pixels as vegetation than the thresholding of the NDVI images.

Figure 25. Pseudo Correctness for R, B and G Channel Based NDVI Veg. Identification.

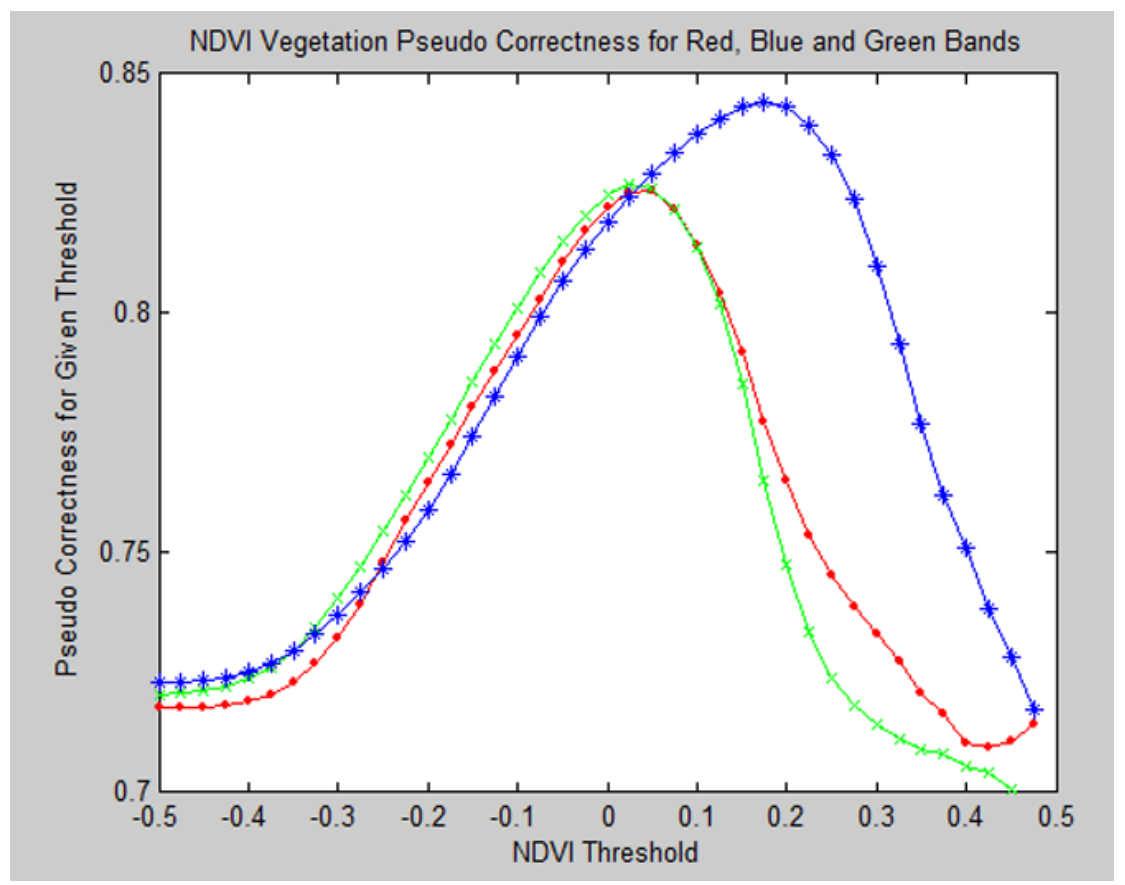


Figure 26. Percentage of Pixels labeled Veg. for Red, Blue and Green Channel Based.

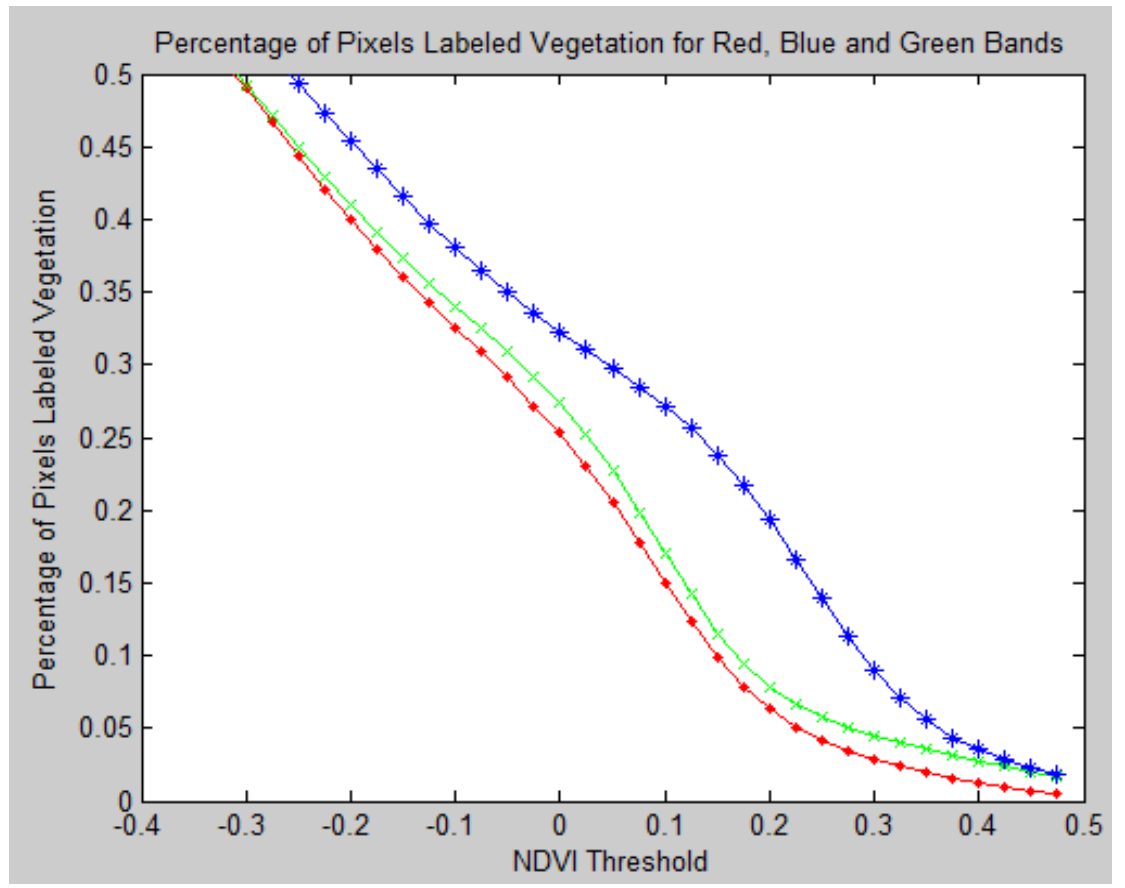

Table 3. Correctness and Coverage Comparison.

\begin{tabular}{lcrl}
\hline Method & Correctness & Coverage & Data Set \\
\hline NDVI Red & $82.55 \%$ & $20.56 \%$ & Fairfield \\
NDVI Green & $82.71 \%$ & $25.23 \%$ & Fairfield \\
NDVI Blue & $84.39 \%$ & $21.67 \%$ & Fairfield \\
Clr Inv Veg & $97.25 \%$ & $30.05 \%$ & Fairfield \\
Clr Inv Veg & $96.91 \%$ & $17.88 \%$ & Anchorage \\
\hline
\end{tabular}

\subsection{Building Detection Comparison with Other Surveyed Methods}

Several approaches in the literature only benchmark their algorithm on less than 250 buildings. The only approach we found tested on more than 250 buildings and reported their building completeness realized a $63.60 \%$. All of the approaches surveyed either didn't report their pixel or their building level correctness; we are the only ones to report both. We have provided in Table 4 a comparison of all the approaches surveyed. The characteristics listed for each approach are "Pix Comp", "Pix Corr", "Bld Comp", "Bld Corr", "\# Bldings", "Resolution", and "Color/Pan" which corresponds to pixel level completeness, pixel level correctness, building completeness, building correctness, the number of buildings the results were based upon, the resolution of the image the algorithm was tested on, and whether that image was panchromatic or color, respectively. The approaches compared are Lefevere et al. [7], Muller et al. [8], Persson et al. [9], Sirmacek et al. [10], Liu et al. [13], and Benediktsson et al. [14]. Also our approach for all buildings ("Shorter all"), then our approach for buildings having an area of $50 \mathrm{~m}^{2}$ and greater (Shorter 50), and finally our approach for buildings 
having an area of $210 \mathrm{~m}^{2}$ and greater (Shorter 210), is also provided. The cells with ' $\mathrm{X}$ 's correspond to data that was not available or not reported. No definitive conclusions are drawn as the data sets used by other authors were different than the data sets we used.

Table 4. Completeness/Correctness at Pixel and Building Level for various Approaches.

\begin{tabular}{lccccccc}
\hline Approach & Pix Comp & Pix Corr & Bld Com & Bld Corr & \#Bldings & Resolution & Color/Pan \\
\hline Lefevre & $63.6 \%$ & $79.4 \%$ & $\mathrm{X}$ & $\mathrm{X}$ & $\mathrm{X}$ & $70 \mathrm{~cm}$ & Pan \\
Muller & $77.3 \%$ & $79.5 \%$ & $\mathrm{X}$ & $\mathrm{X}$ & 240 & $30 \mathrm{~cm}$ & RGB \\
Persson & $53.0 \%$ & $93.0 \%$ & $82.0 \%$ & $\mathrm{X}$ & 17 & $25 \mathrm{~cm}$ & RGB \\
Sirmacek & $\mathrm{X}$ & $\mathrm{X}$ & $86.6 \%$ & $\mathrm{X}$ & 177 & $30 \mathrm{~cm}$ & RGB \\
Liu & $\mathrm{X}$ & $\mathrm{X}$ & $94.5 \%$ & $83.4 \%$ & 277 & $60 \mathrm{~cm}$ & RGB \\
Benediktsson & $80 \%, 95.1 \%$ & $\mathrm{X}$ & $\mathrm{X}$ & $\mathrm{X}$ & 8952,17319 & $1 \mathrm{~m}, 5 \mathrm{~m}$ & Pan, Pan \\
Shorter all & $78.7 \%$ & $51.6 \%$ & $55.4 \%$ & $48.2 \%$ & 2643 & $15 \mathrm{~cm}$ & RGB \\
Shorter 50 & $\mathrm{X}$ & $\mathrm{X}$ & $77.3 \%$ & $64.4 \%$ & 1414 & $15 \mathrm{~cm}$ & RGB \\
Shorter 210 & $\mathrm{X}$ & $\mathrm{X}$ & $91.8 \%$ & $44.5 \%$ & 306 & $15 \mathrm{~cm}$ & RGB \\
\hline
\end{tabular}

\section{Conclusions}

A new method for object classification from a single nadir aerial image is presented. The proposed method uses a novel color quantization technique coupled with a color invariant scheme to identify vegetation. A novel shadow detection procedure is proposed. We use the distance transform coupled with a thresholded entropy filtered image and watershed segmentation to realize entropy segmentation. We then propose the use of solidity as a metric to identify building regions from the entropy segmentation technique. We implemented the proposed method using Matlab and executed the algorithm on an Intel Core 2 Duo $(3.0 \mathrm{GHz})$ machine. The Fairfield data set is a 13,340 $\times 13,340$ pixel image and the Anchorage a 10,896 $\times 10,896$ pixel image. It took the algorithm 90 minutes to complete its execution on the Fairfield data set and 55 minutes for the Anchorage data set.

The method has several advantages which make it attractive for unsupervised building detection from aerial imagery. One important feature is the algorithm is automatic, meaning that there is no user input or parameter adjustment. The only assumptions employed by the algorithm in regards to building structures are that no shadows are cast upon the tops of buildings and that the building rooftop segments exist as convex hulls. The limited assumptions made about buildings enable the proposed algorithm to detect a variety of buildings exhibiting different spectral and structural characteristics. The algorithm is a tertiary classifier capable of classifying objects as non-building and shadow (which shadow assumed to be non-building), building, and vegetation. The proposed vegetation detection realizes approximately $97 \%$ correctness on accurately identifying non-building pixels (as building and vegetation are mutually exclusive classes) across two different data sets. The method only requires a single nadir aerial image for its input. Experiments with real data and comparisons with other proposed methods have shown that the proposed algorithm has competitive performance, despite being completely unsupervised while not implementing overly restricting assumptions about building structures (such as buildings having only red roof tops or having rectangular exteriors). 
The proposed method has some weaknesses as well. Due to the fact that the method is automatic, works off of only a single nadir aerial image, and does not make overly restricting assumptions about building structures, its correctness measure is mediocre. However, it should be noted it is difficult to comparatively rate the correctness measure, both on the pixel and building level as all methods surveyed in this paper only reported one or the other but not both.

\section{Future Work}

Without height information and purely based on luminescence, the classification of concrete surfaces such as roads, sidewalks, parking lots, and buildings becomes difficult - especially when those parking lots, sidewalks and roads exist in the sun-side of buildings. We therefore plan to make use of the height information in the LiDAR data, by registering the two sources of data together, to more accurately distinguish concrete surfaces as building or non-building. Furthermore, other research will be done to remove several of the hardcoded parameters and to test the algorithm on additional data sets.

\section{Acknowledgements}

The authors would like to thank Harris Corporation for funding this research effort; Simon Clode, Franz Rottensteiner and AAMHatch for the procurement and donation of the Fairfield data set and Mr. John Ellis and Aerometric for the Anchorage data set. The authors would also like to thank the anonymous reviewers for their feedback.

\section{References and Notes}

1. Mayer, H. Automatic object extraction from aerial imagery - a survey focusing on buildings. Comput. Vis. Image. Und. 1999, 74, 138-149.

2. Rottensteiner, F.; Trinder, J.; Clode, S.; Kubik, K. Using the dempster-shafer method for fusion of LIDAR data and multi-spectral images for building detection. Inform. Fusion 2005, 6, 283-300.

3. Rottensteiner, F.; Trinder, J.; Clode, S.; Kubik, K. Building detection by fusion of airborne laser scanner data and multi-spectral images: performance evaluation and sensitivity analysis. ISPRS J. Photogramm. 2007, 62, 135-149.

4. Vosselman, G.; Gorte, B.G.H.; Sithole, G. Change detection for updating medium scale maps using laser altimetry. International Archives of Photogrammetry, Remote Sensing and Spatial Information Sciences, Istanbul, Turkey, July 12-23, 2004; pp. 207-212.

5. Aksoy, S.; Ozdemir, B.; Eckert, S.; Kayitakire, F.; Pesarasi, M.; Aytekin, O.; Borel, C.C.; Cech, J.; Christophe, E.; Duzgun, S.; Erener, A.; Ertugay, K.; Hussain, E.; Inglada, J.; Lefevre, S.; Ok, O.; San, D.K.; Sara, R.; Jie, S.; Soman, J.; Ulusoy, I.; Witz, R. Performance evaluation of building detection and digital surface model extraction algorithms: outcomes of the PRRS 2008 Algorithm Performance Contest. 2008 International Association of Pattern Recognition Workshop on Pattern Recognition in Remote Sensing (PRRS 2008), Tampa, FL, USA, December 7, 2008; pp. 1-12. 
6. Jin, X.; Davis, C.H. Automated building extraction from high-resolution satellite imagery in urban areas using structural, contextual, and spectral information. EURASIP J. Adv. Sig. Pr. 2005, 14, 2192-2206.

7. Lefevre, S.; Weber, J.; Sheeren, D. Automatic Building Extraction in VHR Images Using Advanced Morphological Operators. Urban Remote Sensing Joint Event, Paris, France, April 11-13, 2007; pp. 1-5.

8. Muller, S.; Zaum, D. Robust Building Detection in Aerial Images. International Archives of Photogrammetry, Remote Sensing and Spatial Information Sciences, Vienna, Austria, August 29-30, 2005; Vol. 36.

9. Persson, M.; Sandvall, M.; Duckett, T. Automatic Building Detection from Aerial Images for Mobile Robot Mapping. Proceedings 2005 IEEE International Symposium on In Computational Intelligence in Robotics and Automation, Espoo, Finland, June 27-30, 2005; pp. 273-278.

10. Sirmacek, B.; Unsalan, C. Building Detection from Aerial Images Using Invariant Color Features and Shadow Information. In 23rd International Symposium on Computer and Information Sciences, Istanbul, Turkey, October 27-29, 2008; p. 105.

11. Gevers, T.; Smeulders, A.W.M. PicToSeek: combining color and shape invariant features for image retrieval. IEEE Trans. Image Process. 2000, 9, 102-119.

12. Otsu, N. A threshold selection method from Gray-Level histograms. IEEE T. Syst. Man. Cyb. 1979, 9, 62-66.

13. Liu, W.; Prinet, V. Building detection from high-resolution satellite image using probability model, Proceedings 2005 IEEE International Geoscience and Remote Sensing Symposium, 2005. IGARSS '05., Seoul, South Korea, July 24-29, 2005; pp. 3888-3891.

14. Benediktsson, J.A.; Pesaresi, M.; Amason, K. Classification and feature extraction for remote sensing images from urban areas based on morphological transformations. IEEE T. Geosci. Remote. 2003, 41, 1940-1949.

15. Unsalan, C.; Boyer, K.L. A system to detect houses and residential street networks in multispectral satellite images. Comput. Vis. Image. Und. 2005, 98, 423-461.

16. Mathworks Texture Segmentation Using Texture Filters. Available online: http://www.mathworks.com/products/image/demos.html?file=/products/demos/shipping/images/ ipextexturefilter.html (accessed on May 25, 2009).

17. Gonzalez, R.C.; Woods, R.E.; Eddins, S.L. Digital Image Processing Using MATLAB; Prentice Hall: Upper Saddle River, NJ, USA, 2003.

18. Meyer, F. Topographic distance and watershed lines. Signal Process. 1994, 38, 113-125.

19. Image Processing Toolbox-regionprops. Available online: http://www.mathworks.com/ access/helpdesk/help/toolbox/images/index.html?/access/helpdesk/help/toolbox/images/regionpro ps.html (accessed on September 1, 2009)

20. Heipke, C.; Mayer, H.; Wiedemann, C.; Jamet, O. Evaluation of automatic road extraction. Int. Arch. Photogramm. Remote Sens. 1997, 32, 47-56. 
21. Rottensteiner, F.; Trinder, J.; Clode, S.; Kubik, K. Building Detection Using LiDAR Data and Multispectral Images. Digital Image Computing: Techniques and Applications, Sydney, Australia, December 10-12, 2003; Vol. 2, pp. 673-682.

22. NAHB National Association of Home Builders. Rapid Changes Forseen for Home of the Future. Available online: http://www.nahb.org/news_details.aspx?newsID=2886 (accessed on May 12, 2009).

(C) 2009 by the authors; licensee Molecular Diversity Preservation International, Basel, Switzerland. This article is an open-access article distributed under the terms and conditions of the Creative Commons Attribution license (http://creativecommons.org/licenses/by/3.0/). 